\title{
Time-Efficiency of Sorting Chironomidae Surface-Floating Pupal Exuviae Samples from Urban Trout Streams in Northeast Minnesota, USA
}

\author{
Alyssa M. Anderson' and Leonard C. Ferrington Jr'
}

\begin{abstract}
Anderson AM and Ferrington Jr LC. 2012. Time-Efficiency of Sorting Chironomidae Surface-Floating Pupal Exuviae Samples from Urban Trout Streams in Northeast Minnesota, USA. Fauna norvegica 31: $19-23$.
\end{abstract}

Collections of Chironomidae surface-floating pupal exuviae (SFPE) provide an effective means of assessing water quality in streams. Although not widely used in the United States, the technique is not new and has been shown to be more cost-efficient than traditional dip-net sampling techniques in organically enriched stream in an urban landscape. The intent of this research was to document the efficiency of sorting SFPE samples relative to dip-net samples in trout streams with catchments varying in amount of urbanization and differences in impervious surface. Samples of both SFPE and dip-nets were collected from 17 sample sites located on 12 trout streams in Duluth, MN, USA. We quantified time needed to sort subsamples of 100 macroinvertebrates from dip-net samples, and less than or greater than 100 chironomid exuviae from SFPE samples. For larger samples of SFPE, the time required to subsample up to 300 exuviae was also recorded. The average time to sort subsamples of 100 specimens was 22.5 minutes for SFPE samples, compared to 32.7 minutes for 100 macroinvertebrates in dip-net samples. Average time to sort up to 300 exuviae was 37.7 minutes. These results indicate that sorting SFPE samples is more time-efficient than traditional dip-net techniques in trout streams with varying catchment characteristics.

doi: 10.5324/fn.v31i0.1380. Received: 2011-10-14. Accepted: 2012-05-15.

Published on paper and online: 2012-10-17.

Keywords: Chironomidae, pupal exuviae, water quality, trout streams, dip-net

1. University of Minnesota, Department of Entomology, 219 Hodson Hall, 1980 Folwell Avenue, Saint Paul, Minnesota 55108, USA

Corresponding author: Alyssa M. Anderson

E-mail: ande8267@umn.edu

\section{INTRODUCTION}

The Chironomidae are among the most widespread and diverse families of aquatic insects, with representative species exploiting a wide range of microhabitat and water quality conditions (Ferrington et al. 2008). Unfortunately, due to the immense diversity of this family and the relative difficulty and time requirements involved in identification of the larval life stage, the Chironomidae are often ignored in water quality studies, or lumped at family, subfamily or tribe, which does not take into account generic or species-specific pollution tolerance levels (Wilson and McGill 1977; Pinder 1983; Ferrington et al. 2008).

The use of Chironomidae surface-floating pupal exuviae
(SFPE) provides an effective measure of water quality and species richness, and can be used to circumvent many of the inherent problems with benthic samples that can contain large numbers of chironomid larvae. Notably, as compared to benthic samples, the use of chironomid SFPE greatly enhances taxonomic resolution and ease of generic or specieslevel identification, increases the number of taxa collected, allows for collection from a range of habitats, and does not require that stream substrates be disturbed (Ferrington et al. 2008). Thienemann first suggested the use of chironomid pupal exuviae (1910) and the technique has since become widely used in Europe for biomonitoring studies (e.g. Wilson and Bright 1973; Wilson and McGill 1977, 1979; Ruse 1995, 
2002, 2010). The technique has not yet been widely used in North America for biological monitoring, but has proven to be quite useful for a variety of other ecological studies including resolving phenologies (Coffman 1973; Bouchard 2007), determining community composition (Bouchard 2007; Ferrington 2000, 2007; Anderson et al. 2011), quantifying microbial decomposition (Kavenaugh 1988), documenting patterns of diel emergence (Coffman 1974; Wartinbee 1979), and rapid bioassessments of point-source pollution (Ferrington and Crisp 1989; Hayford and Ferrington 2005).

Ferrington et al. (1991) demonstrated the efficiency of using SFPE methodology in an organically enriched stream in an urban area of Kansas, documenting that samples of chironomid SFPE took approximately 30\% less time to sort than traditional dip-net samples for larvae. No other published studies document the time-efficiency of the SFPE sampling technique more broadly across other water quality conditions. The purpose of this study, therefore, was to determine the timeefficiency of processing Chironomidae SFPE samples from urban trout streams compared to dip-net samples.

\section{MATERIAL AND METHODS}

Chironomid SFPE were collected at 17 sample locations across 12 urban trout streams located in or near Duluth, Minnesota, USA. Streams were selected to represent a wide array of catchment sizes and land use conditions. However, water and habitat quality in all streams in this study exceed the conditions of the urban stream in Kansas used by Ferrington et al. (1991) and provide a contrast for testing time-efficiency of processing chironomid SFPE samples. Stream catchments varied from 3.9 square kilometers to 92.2 square kilometers, and urban land use varied from less than $2 \%$ to more than $60 \%$. Substrate conditions of individual streams ranged from predominantly large cobble and boulders interspersed with bedrock outcroppings, to coarse sand. More details of catchment and land use characteristics are available at: http://www.lakesuperiorstreams.org/streams/ troutstreams.html

Samples were collected from each stream on approximately a monthly basis from July through October of 2009, resulting in a total of 68 samples. Of these, 34 samples contained 100 or more chironomid pupal exuviae. Field collections of SFPE followed methods outlined in Ferrington et al. (1991). Briefly, SFPE were collected by dipping a white enamel pan into areas of known SFPE accumulation, such as areas of the stream with foam and/or debris. Contents from the pan were then poured through a $125 \mu \mathrm{m}$ sieve to retain detritus and exuviae. This process was repeated for 10 minutes, working from downstream to upstream. Sieve contents were then preserved in $70 \%$ ethanol.

Dip-net field samples of benthic macroinvertebrates were simultaneously taken during the August and October SFPE collection periods at each of the 17 sample locations, resulting in a total of 34 samples. Of these, 22 samples contained 100 or more invertebrates. Standard multi-habitat sampling procedures were used following protocols outlined by the Minnesota Pollution Control Agency (Chirhart 2003). For each sample, 10 sampling efforts from multiple habitats within a $100-\mathrm{m}$ reach of stream were composited and preserved.

In the laboratory, random subsamples of up to 300 SFPE and 100 macroinvertebrates were picked under $12 \mathrm{X}$ magnification from each composite sample. No restriction was placed on specimen size, nor was any preference given to certain taxonomic groups; the size of the smallest individuals in our samples was $\sim 1 \mathrm{~mm}$. Times (in minutes) needed to pick the specified number of individuals from each sample were recorded. To avoid individual variation in sorting times that might be associated with multiple laboratory technicians with varying levels of experience, all laboratory work reported here was completed by the first author using standardized laboratory protocols. A two-way t-test was then used to test for the difference between mean sort times for 100-count subsamples of dip-net and SFPE samples collected on the corresponding dates in August and October.

\section{RESULTS}

The mean amount of time to sort 100-count subsamples of SFPE was significantly less than required to sort 100-count dipnet samples. Dip-net samples took, on average, 10.3 minutes longer to sort than SFPE samples (32.7 min vs. $21.7 \mathrm{~min}$ ) (p $=0.004 ; 95 \% \mathrm{CI}=3.55-16.97 \mathrm{~min}$ ) (Table 1). The average amount of extra time needed to sort an extra 200 SFPE (from a large sample containing more than 300 specimens), to achieve a subsample total of 300 SFPE was 32.1 minutes. Notably, the maximum and minimum sort times for SFPE samples (12.0 and 57.0 minutes respectively) were also lower than for dip-net samples (22.0 and 70.0 minutes respectively), and exhibited much less variability in total sort time (Figure 1).

In contrast, the mean amount of time required to sort a sample containing less than 100 specimens was 36.5 minutes. Samples that contained from 101 specimens to 299 specimens required 56.4 minutes to sort. The sort times for both of these categories were least efficient in terms of specimens yielded per unit timed effort, and the individual sort times were strongly influenced by the amount of detritus present in the sample, therefore, it would be desirable to characterize the substrate composition in benthic and SFPE samples. The scope of this project did not include these methods, however, future studies are planned to address these issues.

\section{DISCUSSION}

Our results indicate that sorting samples containing large numbers of Chironomidae SFPE from trout streams with 
Table I. Average time in minutes needed to achieve various dip-net and SFPE sample-sorting end-points, indicating replication (N) and standard error of the mean (SEM).

\begin{tabular}{lcclc}
\hline Sampling Method & N & SEM & Activity & Time (min) \\
\hline Dip-Net & 22 & 2.81 & $\begin{array}{l}\text { Average time to sort a dip net sample of 100 macroinvertebrate specimens when } \\
\text { sample has more than 100 specimens }\end{array}$ & 32.7 \\
SFPE & 22 & 1.63 & Average time to sort first 100 SFPE when sample has more than 100 specimens & 21.7 \\
SFPE & 31 & 3.05 & Average time to sort a pupal exuviae sample of less than 100 total specimens & 36.5 \\
SFPE & 17 & 2.38 & $\begin{array}{l}\text { Average time to sort 101 to 300 specimens when a large sample is subsampled } \\
\text { to 300 }\end{array}$ & 32.1 \\
SFPE & 5 & 6.95 & Average time to sort a sample with more than 100 but less than 300 specimens & 56.4 \\
SFPE & 17 & 3.50 & Average time to sort a subsample of 300 SFPE specimens & 51.9 \\
\hline
\end{tabular}



Figure I. Average time needed to sort 100-count dip-net and SFPE subsamples, with $95 \%$ CI for the mean.

varying degrees of urbanization is more time-efficient than sorting dip-net samples of the entire macroinvertebrate community. These results correspond well with those presented by Ferrington et al. (1991) in tests completed at a single sample site in a highly urbanized stream in Kansas that was stressed by organic enrichment from a waste-water treatment plant. The streams used in our study were selected to represent a wide array of urbanization, but collectively have better water quality and higher habitat heterogeneity than the streams in Kansas. Consequently, we feel that the results reported here suggest that collections of SFPE are likely to be more time-efficient across a wide range of water quality conditions, and can represent a cost savings if integrated into large-scale biological monitoring programs.

In addition to the time saved when sorting SFPE samples compared to traditional dip-net samples, the SFPE technique offers various other advantages that are appropriate for largescale biological monitoring programs. SFPE samples can be relatively easily sorted to genus using a dissecting microscope under low magnification. If slide mounted and inspected under higher magnification, accurate species-level identifications should be achievable for many genera. This should be more efficient than working with larvae, where complications with earlier larval instars make it difficult to estimate the number of species in samples (and thus removes the decision of having to choose between the time-consuming slide mounting step for identifying larvae versus lumping all taxa together into family, subfamily or tribe groupings). Furthermore, large numbers of SFPE can be collected in a short amount of time, with these samples representing species residing as larvae in a wide array of microhabitats, some of which might be missed or under-represented in a dip-net sample. Collections may also provide life-stage associations which can aid in identification, particularly if a goal of the project is to identify and describe any new species that might be found.

Bouchard and Ferrington (2010) found that a SFPE subsample size of 100 individuals accounted for approximately $64 \%$ of the species in the chironomid community in trout streams in southern Minnesota. Adding an additional 100 specimens, for a subsample total of 200 taxa, increased the percent total by 13$16 \%$. Increasing the subsample size to 300 resulted in a sample that was sufficiently large enough to collect, on average, $85 \%$ of the total taxa richness. Further, Bouchard and Ferrington (2010) found that when subsampling at the 300-count level, a four-week sampling interval from April-September included, on average, $71 \%$ of the chironomid taxa present. These data, combined with data presented in our work here, will hopefully provide convincing evidence of the efficiency and effectiveness of using SFPE as a biomonitoring tool and encourage more workers and researchers to implement SFPE as a commonly used tool for projects of various scopes and scales.

Our results, however, demonstrate that the number of exuviae collected in a sample can strongly influence the costeffectiveness of subsampling to 100 or 300 specimens. For example, very small samples (i.e. when the number of exuviae is less than the 100-count subsample target) are much more time consuming to sort per exuviae than are larger samples. If a target of 300-count subsample is used, then samples that contain very large numbers of exuviae are most cost-effective to sort per exuviae. When using a 300-count subsample as a target, samples of intermediate size (i.e., greater than 100 but less than 300 specimens) are very expensive per exuviae to process. The numbers of exuviae collected per sample are related to both 
the actual magnitude of emergence immediately before the sample is collected, and the amount of time spent collecting the exuviae. In this study we used the time recommended by Ferrington et al. (1991) to standardize our field efforts across all sample sites and collection dates. However, if the intent of a field sampling protocol is to maximize the cost-per-exuviae collected, our results strongly suggest that samples with larger numbers of exuviae are more efficient, and sampling effort in the field should be adjusted to ensure that a sample contains a large number of exuviae. This could be achieved by extending the time spent sampling to 20 or 30 minutes per site and/or increasing the length of stream used as the sampling reach.

Future steps that will be taken to further justify the use of the SFPE method will be to determine the efficiency of SFPE sample identification time as compared to dip-net samples. Very preliminary data suggest that the time needed to sort and identify 100-count SFPE subsamples to genus is less than that of 100-count dip-net subsamples (e.g., our preliminary values, based on identification times needed by the authors of this manuscript, are 96.6 minutes as compared to 109.2 minutes for SFPE and dip-net samples, respectively), however we do not feel that at this time we have a large enough dataset to make appropriate statistical comparisons that will allow us to draw firm conclusions to this question. Once additional data are generated, it will enable us to draw conclusions as to whether the two sampling methods provide similar estimates of differential levels of stress related to differing patterns of urbanization and impervious substrates in trout streams. Further, once these data are generated, we should then be able to make stronger statements regarding whether SFPE will serve as a viable and cost-effective substitute for more traditional sampling techniques or if we need to find the most effective combination of techniques in order to provide robust results for overall health of a system.

Another variable that influences the sorting time of samples is the amount of detritus and fine sediments collected along with the exuviae. The streams used for this study had very little fine particulate organic matter in suspension, and very little was inadvertently accumulated into the samples. However, small twigs and other floating coarse detritus (such as shed flower parts, seeds, deciduous leaves, and small cones of conifers) were occasionally abundant and likely contributed appreciably to the sort times of samples with more than 100 but less than 300 specimens. To refine our estimates of sorting efficiencies, we are in the process of developing a standardized method for quantifying the fraction of a sample that consists of fine versus more coarse particulate matter, which we will use to further refine our estimates in future studies.

\section{ACKNOWLEDGMENTS}

We wish to thank Petra Kranzfelder, Frances Matos-Schultz, Nathan Roberts, and Kimerly Wilcox for their assistance with field research. Thanks also to Blair Bolig and Katrina Shaw for their help in the lab. Funding for this project was provided by the Minnesota SeaGrants Program.

\section{REFERENCES}

Anderson AM, Bouchard RW, Ferrington LC. 2011. Hibernal emergence of Chironomidae (Diptera) in relation to stream size in Kansas, USA. In: Wang X, Liu W. (eds). Contemporary Chironomid Studies: Proceedings of the $17^{\text {th }}$ International Symposium on Chironomidae. Nankai University Press. pp. 247-255

Bouchard RW. 2007. Phenology and taxonomic composition of lotic Chironomidae (Diptera) communities in contrasting thermal regimes. Doctoral Thesis, University of Minnesota. $411 \mathrm{p}$.

Bouchard RW, Ferrington LC. 2010. The effects of subsampling and sampling frequency on the use of surface-floating pupal exuviae to measure Chironomidae (Diptera) communities in wadeable temperate streams. Environmental Monitoring and Assessment 181: 205-223.

Chirhart J. 2003. Development of a macroinvertebrate index of biological integrity (IBI) for rivers and streams of the Saint Croix River Basin in Minnesota. Publication of the Minnesota Pollution Control Agency, Saint Paul, MN. 41 p.

Coffman WP. 1973. Energy flow in a woodland stream ecosystem. II. The taxonomic composition and phenology of the Chironomidae as determined by the collection of pupal exuviae. Archiv für Hydrobiologie 71: 281-322.

Coffman WP. 1974. Seasonal differences in the diel emergence of a lotic chironomid community. Entomologisk Tidskrift 95: 42-48.

Ferrington LC. 2000. Hibernal emergence patterns of Chironomidae in lotic habitats of Kansas versus ambient air and water temperatures. In: Hoffrichter O. (ed.). Late 20th Century Research on Chironomidae. An Anthology from the 13th International Symposium on Chironomidae, 5-9 September 1997. Shaker Verlag, Aachen. pp. 375-382.

Ferrington LC. 2007. Hibernal emergence patterns of Chironomidae in lotic habitats of Kansas versus substrate composition. In: Andersen T. (ed.). Contributions to the Systematics and Ecology of Aquatic Diptera - A Tribute to Ole A. Sæther. The Caddis Press, Columbus. pp. 99-105.

Ferrington LC, Blackwood MA, Wright CA, Crisp NH, Kavanaugh JL, Schmidt FJ. 1991. A protocol for using surface-floating pupal exuviae of Chironomidae for rapid bioassessment of changing water quality. In: Peters NE, Walling DE. (eds). Sediment and stream water quality in a changing environment: trends and explanation. IAHS Publication 203: 181-190. IAHS Press, Oxfordshire, UK.

Ferrington LC, Coffman WP, Berg MB. 2008. Chironomidae. In: Merritt RW, Cummins KW, Berg MB. (eds). An Introduction to the Aquatic Insects of North America. Kendall/Hunt, Dubuque, IA. pp. 847-1003.

Ferrington LC, Crisp NH. 1989. Water chemistry characteristics of receiving streams and the occurrence of Chironomus riparius and other Chironomidae in Kansas. Acta Biologia Debrecina, 
Oecologica Hungarica 3: 115-126.

Hayford BL, Ferrington LC, Jr. 2005. Biological assessment of Cannon Creek, Missouri by use of emerging Chironomidae (Insecta: Diptera). Journal of the Kansas Entomological Society 78: 89-99.

Kavanaugh RG. 1988. Decomposition studies of Chironomidae pupal exuviae (Chironomidae: Diptera). Master's Thesis, University of Kansas, Lawrence. 104 p.

Pinder LCV. 1983. The larvae of Chironomidae (Diptera) of the Holarctic region - Introduction. Entomologica Scandinavica Supplement 19: 7-10.

Ruse L. 1995. Chironomid community structure deduced from larvae and pupal exuviae of a chalk stream. Hydrobiologia 315: 135-142.

Ruse L. 2002. Chironomid pupal exuviae as indicators of lake status. Archiv für Hydrobiologie 153: 367-390.

Ruse L. 2010. Lake reference state deduced from chironomid pupal skin data. In: Ferrington LC. (ed.). Proceedings from the XV International Symposium on Chironomidae, August 2003. University of Minnesota Press. pp. 140-155.

Thienemann A. 1910: Das Sammeln von Puppenhäuten der Chironomiden. Archiv für Hydrobiologie 6: 213-214.

Wartinbee DC. 1979. Diel emergence patterns of lotic Chironomidae. Freshwater Biology 9: 147-156.

Wilson RS, Mcgill JD. 1977. A new method of monitoring water quality in a stream receiving sewage effluent, using chironomid pupal exuviae. Water Research 11: 959-962.

Wilson RS, Mcgill JD. 1979. The use of chironomid pupal exuviae for biological surveillance of water quality. Department of the Environment, Water Data Unit, Technical Memorandum 18. $20 \mathrm{p}$.

Wilson RS, Bright PL. 1973. The use of chironomid pupal exuviae for characterizing streams. Freshwater Biology 3: 283-302. 